\title{
Inversiones e intermediaciones financieras en el mercado del suelo urbano. Principales hallazgos a partir del estudio de transacciones de terrenos en Santiago de Chile, 2010-2015
}

Ivo Gasic. Universidad Alberto Hurtado, Santiago, Chile.

RESUMEN | Se caracterizan los capitales que convergen en el mercado del suelo del Área Metropolitana de Santiago, planteando que bajo procesos de financiarización de la economía capitalista mundial y su penetración a nivel nacional, es previsible una mayor colocación de capitales de origen financiero en la adquisición de terrenos para desarrollo inmobiliario. Para estudiar dicho proceso se emplea un método de investigación empírica de análisis de transacciones de terrenos. Se utiliza una base de datos de transacciones registradas en los conservadores de bienes raíces del Área Metropolitana de Santiago para el período 2010-2015. El principal resultado obtenido es que, en dicho período, los mayores compradores de terrenos corresponden a entidades financieras representadas por sociedades de inversión, bancos y compañías de seguros de vida. Las adquisiciones de entidades financieras superan incluso la participación del conjunto de sociedades del rubro inmobiliario, lo que revela la importancia que adquieren las lógicas de valorización del capital financiero en el mercado del suelo urbano.

PALABRAS ClaVe | economía urbana, mercado del suelo, mercado inmobiliario.

ABSTRACT | The capitals that converge in the Santiago's urban land market have been characterized in this work. It is considered that under financialization processes of the world capitalist economy and its penetration at the national level, it is foreseeable a greater placement of capital of financial origin that are available to the land positioning to expand the metropolitan real estate activity. An empirical method of land transaction analysis is used to study this process, with a database of transactions registered in the Metropolitan Santiago's Area for the period 2010-2015. The main result obtained is that, during this period, the largest buyers of land correspond to financial entities represented by investment companies, banks and insurance companies. The acquisitions of financial institutions exceed even the participation of all companies in the real estate sector, which reveals the importance of the logic of valorization of financial capital in the urban land market.

KEYWORDs | urban economy, land market, real estate market.

Recibido el 28 de diciembre de 2016, aprobado el 27 de febrero de 2017

E-mail: igasic@uahurtado.cl 


\section{Introducción}

El Área Metropolitana de Santiago es un laboratorio envidiable para los investigadores interesados en observar los múltiples canales por los que circula el capital financiero dentro del sector inmobiliario y de desarrollo urbano. Por una parte, se percibe un incremento notable de la producción inmobiliaria, estimulada por la articulación de promotores de vivienda con entidades financieras, desde bancos hasta compañías de seguros y fondos de inversión. Por otra, existen altos niveles de endeudamiento de los hogares por adquisición de vivienda en propiedad vía crédito, sea para uso propio o para renta, donde destaca el protagonismo coyuntural del segmento de pequeños inversionistas. Tanto por el lado de la oferta como de la demanda se han ido diversificando los agentes e instrumentos financieros, lo que ha determinado el ritmo de crecimiento del sector inmobiliario y, a su vez, ha contribuido a transformaciones radicales del medio urbano construido de Santiago (Cattaneo, 2011; Daher, 2013; De Mattos, 2013, 2016).

En Chile, en general, los estudios sobre esta materia se han enfocado en la diversificación de las formas de financiamiento a la demanda y a los desarrolladores inmobiliarios, así como en la creación de fondos y sociedades ad hoc a la formación de conglomerados financiero-inmobiliarios (Alarcón, Demaestri \& Piedrabuena, 2014; Cattaneo, 2011; Daher, 2013; De Mattos, 2013). Sin embargo, se ha prestado poca atención a las formas de financiarización del suelo urbano. Los pocos estudios del mercado del suelo en Santiago han tenido su foco en la formación de precios hedónicos y en el modelamiento geoestadístico del mercado, concentrando la discusión siempre en las variables de precio, disponibilidad y oferta. Pero poco se ha avanzado en el conocimiento de las instituciones y agentes financieros que han ido estructurando el mercado del suelo, lo que podría ser una veta de exploración sobre las dinámicas de financiarización del desarrollo inmobiliario y urbano.

Frente a la ausencia de investigación empírica sobre este asunto en Chile, el presente trabajo intenta aportar algunos elementos de carácter descriptivo, que han sido ordenados a partir de una pregunta de investigación general. A saber, en una economía financiarizada donde los capitales -financieros- han ingresado a las distintas fases del sector inmobiliario en Chile, ¿qué nivel y forma de participación adquieren las entidades financieras en la incorporación de terrenos para desarrollo inmobiliario? ¿Es posible identificar una lógica financiera en la adquisición directa de terrenos para desarrollo inmobiliario, o las entidades financieras siguen operando a la manera tradicional, con préstamo a los desarrolladores, y, por ende, incidiendo solo indirectamente sobre el mercado del suelo?

Planteada esta pregunta, se ha diseńado una investigación exploratoria de identificación y caracterización de agentes que compran terrenos en Santiago, aportando con una base empírica (no-analítica) que permite enmarcar las posibles explicaciones sobre cómo las entidades financieras valorizan sus capitales en el mercado del suelo. Este artículo expone los principales hallazgos de la investigación, organizando su contenido en cuatro secciones: (i) interpretación general de la liberalización del suelo urbano y la financiarización inmobiliaria en Santiago; (ii) diseño de la investigación; (iii) resultados principales; y (iv) conclusiones y reflexión final. 


\section{La liberalización del suelo urbano y la incorporación capitalista de terrenos para desarrollo inmobiliario en Santiago}

La Política Nacional de Desarrollo Urbano (PNDU) de 1979 ha sido sindicada insistentemente como una política de liberalización del mercado del suelo urbano, donde se definió que el suelo no era un bien escaso y que debían concurrir a él libremente los agentes del mercado, eliminando las restricciones públicas a la oferta. ${ }^{1}$ Sin embargo, la PNDU sería a penas el corolario de un proceso más amplio, no solo de liberalización, sino de fomento a la incorporación de terrenos al circuito de producción inmobiliaria capitalista. De hecho, entre 1974 y 1979, antes de la PNDU, se implementaron diversos cambios regulatorios y de política urbana orientados a facilitar el ingreso de capital de empresas inmobiliarias y de la construcción al mercado del suelo. Destacan en este sentido las medidas tomadas para reducir los impuestos a la tenencia y transacción de tierras, a través de: (i) disminución en la tasa de impuesto de transferencia de bienes raíces desde un 8\% a un 0,5\% (1976-1980); (ii) eliminación de los impuestos a los sitios vacantes (1976); y (iii) ampliación de la exención tributaria a predios residenciales que sean edificados en una superficie de hasta $162 \mathrm{~m}^{2}$ (DFL-2).

De esta forma, a lo largo de 1974-1980 se promovió un proceso de integración del mercado de tierras al desarrollo inmobiliario que tuvo como corolario la promulgación de la PNDU-1979 y la extensión drástica del límite urbano en el mismo año. Es decir, más que el restablecimiento de un libre mercado del suelo, se fomentó la constitución de un sector inmobiliario capitalizado de terrenos, que permitiría el despliegue del conjunto de los capitales del sector de la construcción acumulados en décadas previas. Nótese que Chile atravesó en los años sesenta una fase de explosión del sector de la construcción, en coherencia con un período de pretensiones desarrollistas con fomento a la producción, en este caso de infraestructura y edificaciones, básicamente vivienda, para solventar las fuertes migraciones intranacionales de las décadas cincuenta y sesenta, con énfasis sobre el Área Metropolitana de Santiago. ${ }^{2}$

De esta manera, las políticas de inicios de los ańos sesenta de fomento a la construcción -no necesariamente a la privatización del suelo- permitieron incubar un sector productivo con capacidad de acumulación de capital y representación a nivel nacional, a través de la Cámara Chilena de la Construcción, creada en 1951, orientada a defender los intereses gremiales y con fuerte participación en el diseño de políticas públicas urbanas y económicas (Cheetham, 1971). Esta fase de acumulación en un sector propiamente productivo (1960-1973), propulsado por el Estado y

1 En 1979, vía Decreto 420, se extendió el límite urbano en un 160\% del área urbana a la fecha, abriendo nuevas 60.000 hectáreas sobre la base de 36.000 hectáreas, eliminando prácticamente el límite urbano (Pablo Trivelli \& Cía. Ltda., 2010).

2 Entre 1940 y 1959, la superficie de vivienda autorizada a nivel nacional se mantuvo entre los 500.000 y $800.000 \mathrm{~m}^{2}$, y luego, tras la aplicación del DFL-2 en 1959, se logró más que duplicar la cifra, llegando a los 2 millones de $\mathrm{m}^{2}$ de vivienda autorizados en dicho año. Luego de eso, la construcción de viviendas se mantuvo estable, con fluctuaciones menores, hasta la crisis de 1982 1983, cuando se disparó aceleradamente hasta 1997, llegando a superar los 9 millones de $\mathrm{m}^{2}$ autorizados para vivienda, que cayeron luego abruptamente por la crisis asiática a niveles en torno a los 6 millones de $\mathrm{m}^{2}$ entre 1999 y 2002. 
su gestión inmobiliaria pública, sentó la base para un posterior proceso de apertura a un mercado inmobiliario posibilitado por las reformas urbanas y de liberalización del suelo aplicadas entre 1974 y 1980.

Interpretando así los hechos, es posible construir un marco histórico general que ponga en el centro la relación vertical entre el capital de la construcción y la propiedad de la tierra antes que la relación horizontal de agentes en el mercado. Tomando como base las proposiciones de Sabatini y Donoso (1979, 1980), planteadas más bien como hipótesis o conjeturas precisamente en el período de reforma que se comenta, se pueden plantear las siguientes fases por las que atraviesa la gestión inmobiliaria del suelo en el Área Metropolitana de Santiago del siglo XX: (i) dominancia de propietarios-rentistas en la retención y loteo de predios, hasta la década de 1940; (ii) dominancia de empresas loteadoras especializadas, en período 1940-1960; (iii) dominancia de gestión pública del suelo con políticas de fomento a la actividad privada de construcción, pero no rentista (barreras a las operaciones de loteo, subdivisión y costos de transferencia de propiedades), entre 1960 y 1973; (iv) dominancia de la gestión privada del suelo por parte de los promotores inmobiliarios desde 1973 en adelante, ${ }^{3}$ mostrando alto nivel de integración al sistema bancario y financiero desde la segunda mitad de la década de los ochenta.

Precisamente sobre este último momento lo que se observa es que, desde los ochenta, los capitales de la construcción comienzan a integrar el negocio inmobiliario de una manera más decidida, al amparo de la nueva política urbana y de suelo, pero con cierto apoyo del sector financiero, básicamente bancario. Se torna necesario entonces conocer cómo se desarrolla el binomio inmobiliario-financiero en Chile y, luego, cómo se va desenlazando su cooperación en la incorporación capitalista de terrenos para desarrollo de productos inmobiliarios, básicamente vivienda.

\section{Financiarización inmobiliaria en Chile}

Tras la crisis y reestructuración capitalista de la década de los setenta, la economía mundial ha experimentado un ascenso generalizado del sector financiero, ${ }^{4}$ conformando a su vez un sistema organizado de instituciones y agentes encargados de financiarizar a hogares y empresas del sector productivo (Lapavitsas, 2016). Entre los sectores que han sido mayormente impactados por la financiarización de la economía se encuentra el rubro inmobiliario (Bellamy-Foster, 2010; Daher, 2013; De Mattos, 2016; Harvey, 2012; Hudson, 2010), no solo por la tradicional articulación de la banca con el financiamiento de la demanda habitacional, sino también por

Con posterioridad a 1973, la inversión pública se restringe considerablemente, las reservas estatales de terrenos se liquidan y se liberalizan los cánones de arrendamiento. Estas medidas, vigentes ya al ańo 1975, fueron seguidas por otras con la misma orientación de liberalizar el funcionamiento del mercado de tierras e inmobiliario en general (Sabatini \& Donoso, 1979, p. 16).

4 Entre los principales factores histórico-sociales que explican este fenómeno se encuentran: (i) descenso generalizado de la tasa media de ganancia y de acumulación de capital en los sectores productivos en la mayoría de los países del centro capitalista (Aglietta, 1979; Husson, 2010); (ii) crisis de sobreacumulación de mercancías en algunos sectores de la economía, produciendo fuertes movimientos sectoriales de capital a nivel global y de refugio en activos especulativos (Chesnais, 2003); y (iii) posicionamiento del dólar como moneda internacional (Lapavitsas, 2016). 
la progresiva transformación de inmuebles en activos financieros (Harvey, 1974). Estos activos, por una parte, permiten la realización de operaciones especulativas y, por otra, estimulan al sector de la construcción y urbanización, que ha sido fundamental para la acumulación en la actual fase del capitalismo 5 (Harvey, 2012). En este sentido, más allá de las relaciones teóricas que puedan establecerse, se constata empíricamente la conformación de un conglomerado inmobiliario-financiero en varios países del centro capitalista, lo que ha recibido internacionalmente el nombre de FIRE Sector por su acrónimo en inglés (Financial, Insurance, and Real Estate).

El proceso de financiarización de la economía es particularmente importante en Chile, dado que este es uno de los países periféricos que experimentó mayor reestructuración y posicionamiento en la economía global precisamente en la fase financiarizada del capitalismo. En particular en el sector inmobiliario, el sistema de instituciones y agentes financieros ha penetrado fuertemente a través de distintos mecanismos, los cuales han operado ya sea de forma complementaria o sustitutiva; entre ellos: (i) establecimiento del sistema de pensiones, y particularmente de las Administradores de Fondos de Pensiones (AFP), que bajo el principio de capitalización individual han abierto múltiples canales para transformar el ahorro en capital prestable, incidiendo de forma indirecta en un volumen muy considerable de la producción de vivienda; (ii) creación de los Fondos de Inversión Inmobiliaria, inicialmente pensados para estimular un segmento de pequeños inversionistas; (iii) incremento sustantivo de la participación de las Compañías de Seguros de Vida (csv) en la inversión inmobiliaria directa, además de la compra de letras hipotecarias; y (iv) apertura de empresas inmobiliarias y de la construcción a la bolsa de valores. Todos estos mecanismos han ingresado a diversificar las fuentes de financiamiento inmobiliario tradicionalmente ligadas al sector bancario.

En el caso de las Afp, durante los años ochenta concentraron su participación de manera indirecta en el sector inmobiliario, comprando letras hipotecarias hasta por un $43 \%$ del total de su portafolio, lo que disminuyó en la década de los noventa en torno al 16\%, siendo reemplazadas las letras por vehículos más directos, sofisticados y orientados a la oferta, como son los fondos de inversión inmobiliaria. Creados bajo la Ley $\mathrm{N}^{\circ} 18.815$ de Fondos de Inversión, los fondos inmobiliarios funcionaron inicialmente de manera muy integrada a las AFP, aportando estas con un total de 11.000 millones de pesos al 1993, representativos del 45,5\% de participación en el total de los fondos de inversión inmobiliaria (Daher, 1995). Como señala el informe de la Asociación Gremial de AfP de 2012, los fondos de pensiones han sido uno de los más importantes vehículos de financiamiento al sector inmobiliario desde los noventa hasta la fecha ${ }^{6}$ (Asociación Afp Chile, 2012), lo que dista de ser un caso

5 La burbuja inmobiliaria en EEUU y España en el periodo 2001-2009, así como la urbanización china de las últimas décadas (Fondo Monetario Internacional [FMI], 2015), han demostrado no solo las contradicciones de la especulación financiera, sino también los fuertes vínculos entre desarrollo inmobiliario, urbanización y economía real.

6 "Los Fondos de Pensiones han aportado recursos financieros de largo plazo al mercado de capitales. Uno de los sectores que más se ha beneficiado es el inmobiliario. En efecto, los fondos previsionales poseen el $47 \%$ de las Letras Hipotecarias del mercado, con una inversión cercana a US\$5.400 millones. Con esto se financia la adquisición o construcción de bienes inmuebles, 
excepcional chileno, dada la vasta correspondencia con la financiarización inmobiliaria de los fondos de pensiones en distintos países europeos (Theurillat, 2012).

A pesar de la importante presencia de los inversionistas institucionales, como las AFP, Cattaneo (2011) detecta una segunda oleada de fondos de inversión inmobiliaria entre 2004 y 2008, con mayor presencia de inversionistas particulares. Entre esos años, los fondos inmobiliarios se orientaron al desarrollo de vivienda nueva más que al negocio de la renta, e impulsaron obras por casi 21.000 unidades, es decir, un $8,7 \%$ del total de permisos de edificación emitidos para esos cinco años (Cattaneo, 2011). ${ }^{7}$ Además de los volúmenes de participación, se ha detectado cierta regularidad en la asociación entre determinados desarrolladores y fondos, generando de esta forma un efecto de reorganización del sector inmobiliario a través del acceso a financiamiento por fondos (Cattaneo, 2011).

Por su parte, se ha profundizado la participación de otros inversionistas institucionales que intervienen de forma directa en el desarrollo inmobiliario, a diferencia de las administradoras de pensiones, que han utilizado la mediación de los fondos de inversión. Es el caso de las Compañías de Seguros de Vida, las cuales han registrado un aumento significativo en activos inmobiliarios al menos en los últimos diez años. Las series estadísticas de la Superintendencia de Valores y Seguros (svs) muestran que entre 2005 y 2014 el ítem de "Inversiones Inmobiliarias" se ha posicionado entre los cinco ítems de inversión más importantes - de los quince ítems registrados en los balances de las compañías-, con un monto anual promedio de 101 millones de UF, y cerrando al 2014 con 162 millones de UF, monto superado solamente por la inversión en "Bonos y Deventures", que son largamente los más importantes en el total de las inversiones de las compañías de seguros de vida en el período. ${ }^{8}$

En términos relativos, la participación de las "Inversiones Inmobiliarias" sobre el total de las inversiones es también ilustrativa de la importancia que va adquiriendo el sector inmobiliario en las compañías de seguros de vida en el período 2005-2014. Salvo los fondos mutuos y las cuentas corrientes, que son marginales en participación, se observa que los aumentos más significativos en participación corresponden a inversiones en el exterior (286\% variación), inversiones inmobiliarias $(80 \%)$ y bonos bancarios (68\%), logrando cada uno una participación en torno al 13-14\% en 2014 (svs, 2016). Los datos de la Superintendencia de Valores y Seguros grafican, entonces, que además de los fondos de inversión inmobiliaria, aparecen las compañías de seguros de vida como importantes inversionistas en activos inmobiliarios, que los distingue sustantivamente de las operaciones indirectas que tienen que seguir otros inversionistas institucionales, como las AFP.

principalmente habitacional (...). Además, con una inversión adicional de us\$520 millones en cuotas de Fondos de Inversión Inmobiliarios, los Fondos de Pensiones invierten en otras áreas del negocio inmobiliario, tales como: oficinas, centros comerciales, mutuos hipotecarios, terrenos, financiamiento de proyectos, etc." (AAFP, 2012; en De Mattos, documento interno, 2013).

7 Si a esto se agregan las 10.000 viviendas financiadas con fondos de inversión mixtos, se obtiene para ese período una participación del 13,8\% de las viviendas privadas construidas en Santiago, lo cual supera como conjunto a la cifra de participación de cualquier desarrollador en particular.

8 A esto podría sumársele la participación de las aseguradoras en fondos de inversión y en acciones que eventualmente podrían ser canalizados a las empresas inmobiliarias. 
Otro rasgo importante de un proceso de financiarización, y que se ha venido registrando desde mediados de los 2000 en Chile, es la apertura en bolsa de los principales desarrolladores inmobiliarios y constructoras de vivienda del país: Pazcorp s.A. y Socovesa s.A., Moller y Pérez Cotapos S.A., Salfacorp s.A. y Besalco S.A. ${ }^{9}$ La apertura en bolsa de estas empresas puede considerarse también como parte del proceso de financiarización del desarrollo inmobiliario nacional, en el cual los bancos han dejado de ser el único medio de financiamiento a los principales desarrolladores del país. Al observar los inversionistas que han comprado acciones de estas empresas, Zegers (2014) ha notado la presencia de fondos de inversión, AFP y fondos mutuos, que dan cuenta de nuevos circuitos de capital financiero mediante el mercado bursátil, complejizando la primacía de la banca en el financiamiento al desarrollo inmobiliario.

De lo anteriormente comentado se puede concluir una mayor integración de los capitales financieros e inmobiliarios en Chile, superando la lógica inicial de articulación directa entre los sectores inmobiliario y bancario. Esta financiarización se comprende desde la creación de las AFP a inicios de los ochenta hasta la apertura bursátil de los desarrolladores a mediados de los 2000, pasando por la creación de los distintos fondos de inversión inmobiliaria a fines de los ochenta y la inversión inmobiliaria directa de las compañías de seguros de vida, intensificada en los 2000. Todos estos datos han podido recolectarse a partir de bases estadísticas oficiales, balances de compañías y de publicaciones académicas y estudios sobre la materia. No obstante, la especial opacidad del mercado del suelo y la escasez de investigaciones de base empírica han hecho muy difícil, hasta la fecha, la observación pormenorizada de lo que ocurre con la inversión financiera en terrenos urbanos. Este trabajo pretende llenar ese vacío y proponer conjeturas sobre la lógica subyacente en los principales agentes, entendiendo que esta veta de análisis podría informar bastante sobre un aspecto poco estudiado de la financiarización inmobiliaria.

\section{Diseño de la investigación}

Este trabajo se centra en la identificación y caracterización de los agentes que participan del mercado del suelo urbano en Santiago, observando a su vez el peso relativo de aquellas sociedades y empresas de carácter inmobiliario y financiero, sosteniendo como supuesto teórico una alta intermediación financiera en terrenos para desarrollo (básicamente vivienda nueva) y plusvalía. Para esto se ejecutan tres tareas de investigación: (i) clasificar sectorialmente los capitales que participan del mercado del suelo; (ii) identificar y caracterizar las principales sociedades y empresas que participan del mercado del suelo; y (iii) analizar el tamańo de las sociedades y empresas implicadas, y su nivel de concentración de mercado.

En cuanto al proceso de recolección, el investigador emplea una base de datos obtenidos del Registro de Propiedades en los cuatro conservadores de bienes raíces de

Las tres primeras con marcado énfasis en desarrollo de vivienda, mientras las dos últimas utilizan su capital de construcción en infraestructura e inmobiliario de distinto tipo. 
la Región Metropolitana entre los ańos 2010 y 2015, ${ }^{10}$ aunque en el presente texto se incluyan solo los datos correspondientes al Conservador de Bienes Raíces de Santiago (CBRS, representativo de un 70-75\% del total de transacciones de terrenos a nivel metropolitano). Es necesario indicar que a partir de esta base de datos se ha considerado como terreno toda propiedad transada bajo el rótulo de parcela, lote, sitio o terreno, lo cual indica dos limitaciones metodológicas relevantes: (i) no se contabilizan las propiedades edificadas que son valorizadas como terreno por parte de los compradores, lo que margina una cantidad considerable de propiedades adquiridas para demolición y reedificación en las áreas centrales y pericentrales; (ii) se contabiliza indistintamente a las parcelas de agrado acogidas a la ley de predios rústicos, que representan una proporción muy alta del total de terrenos transados -dado el mercado de parcelas en las comunas periurbanas, sobre todo de Colina y Lampay que, para objetos de este análisis, expresan una dinámica inmobiliaria distinta, menormente mediada por desarrolladores capitalistas y entidades financieras.

El investigador ha cruzado esta base de datos con información del Servicio de Impuestos Internos (sII), generando dos cruces relevantes. Por una parte, un cruce con el Catastro de Predios del siı para los años de inicio y cierre del período de estudio (2010 y 2015), ${ }^{11}$ realizado a través de los roles de los terrenos transados, permitió agregar los siguientes datos claves para el estudio: avalúo fiscal, superficie de terreno, superficie construida (en caso de tener alguna edificación, pese a la categoría de terreno) y destino del bien raíz. Por otra parte, se ha generado un segundo cruce con el Registro de Contribuyentes (RC) del siı al año 2014 (vigente para 2015), a través del RUT de los compradores de terrenos. ${ }^{12}$ Esto permitió identificar a las personas naturales y jurídicas que participan en la compra de terrenos, agregando información relevante de la base de datos RC-siI, tales como razón social, actividad económica, rubro y sub-rubro, capital propio y tramos de facturación.

Sobre el análisis de los datos, los tres objetivos mencionados han sido atendidos de la siguiente manera. Primero se efectuó la categorización de los sectores y actividades económicas dominantes en el mercado, considerando que el objeto de este trabajo es analizar particularmente las operaciones de los desarrolladores inmobiliarios y de los agentes financieros asociados a la gestión inmobiliaria. La categorización se ha realizado según rubro, sub-rubro y actividad económica, obteniendo un alto grado de especificación sectorial, aunque sea solo relativo a la actividad principal de cada contribuyente.

El segundo momento de análisis sobre esta base de datos consta de la identificación particularizada de los principales agentes, empresas y sociedades que invierten

10 Provista por la empresa Transsa Limitada.

11 Tal cruce permite obtener las superficies y destinos de los terrenos, los cuales no podrían obtenerse con una base de datos únicamente basada en datos actuales. Al respecto, lo relevante es saber la superficie predial al momento de la transacción, no aquella que actualmente tiene la propiedad, dado que pueden existir subdivisiones o fusiones que alteren significativamente las superficies, sobre todo tratándose de terrenos incorporados al desarrollo inmobiliario.

12 Este nuevo cruce obedece a que las bases de datos de los conservadores contienen RUT comprador, que corresponde a una exigencia impuesta a los conservadores de bienes raíces desde el ańo 2008, que implica registrar RUT comprador y certificación de contribuciones pagadas. El registro de RUT comprador ha permitido vincular con la base de datos del sir en una proporción importante. 
en adquisición de terrenos en Santiago. Utilizando la misma información antedicha, en este momento de análisis se identifica a las empresas y se estima su volumen de inversión en suelo. Esto permite, por una parte, observar si estos agentes son efectivamente desarrolladores inmobiliarios, o si aparecen otras empresas o sociedades con mayor cantidad y volumen de adquisiciones en terrenos. En definitiva, el objeto es identificar a los agentes dominantes en el mercado del suelo y discutir sobre su posible relación de colaboración, competencia o relación societaria respecto a los principales desarrolladores inmobiliarios de vivienda.

Por último, se analizó el tamaño de las sociedades y empresas implicadas, así como su nivel de concentración de mercado, según la información de tramo de facturación y capital propio positivo. Esta información permite estimar el tamańo de los agentes implicados en estas operaciones y a la vez observar la participación relativa que tienen en este mercado las empresas y sociedades más grandes del país. Al igual que en toda la investigación, el nivel de conocimiento sobre la materia remite a la descripción, clasificación y categorización de datos, intentando con ello nutrir de una base empírica inédita a posteriores trabajos con alcance explicativo propiamente tal.

\section{Resultados}

Entre enero de 2010 y abril de 2015 se han realizado 18.601 transacciones de terrenos, con un flujo anual estabilizado en 3.600 registros. En ocasiones hay más de una transacción por predio en el período, lo cual implica que de las 18.601 transacciones, puede individualizarse un total de 17.879 predios transados según rol de propiedad. Considerando transacciones - no individualización de predios-, se obtiene una superficie total de 11.485 hectáreas transadas entre enero de 2010 y abril de 2015, con una media anual de 2.248 hectáreas. Este es el volumen total de superficie que se mueve por el mercado del suelo en Santiago, lo que no representa un flujo al mercado inmobiliario propiamente, ya que los terrenos no "ingresan al mercado", sino que se mueven dentro de él, pero representa de buena manera la dimensión que adquiere el movimiento mercantil de terrenos, que estaría logrando en cinco años un flujo aproximado equivalente a un 15\% de la superficie del Área Metropolitana de Santiago.

Al cruzar estos datos con el registro de contribuyentes del sil vigente al 2015, se obtuvieron los siguientes resultados: (i) la mayor cantidad de adquisiciones proviene de entidades del rubro de "intermediación financiera"; (ii) dentro del rubro financiero, las entidades que más compran terrenos corresponden a sociedades de inversión altamente fragmentadas; (iii) existe un núcleo de capital financiero altamente centralizado, compuesto por bancos y compañías de seguros de vida, aunque con una participación sustantivamente menor en comparación a las sociedades de inversión; y (iv) las grandes empresas son las que más invierten en el mercado de terrenos de Santiago, representadas por el capital bancario y asegurador. Estos cuatro hallazgos serán detallados a continuación. 
La mayor cantidad de adquisiciones proviene de entidades del rubro de "intermediación financiera"

Al observar los distintos rubros económicos declarados en el sir, se detecta que las sociedades que mayormente compran terrenos en Santiago corresponden al rubro de "intermediación financiera", con un total de 2.765 propiedades, representativas del $40 \%$ de las adquisiciones en el período. Este hallazgo es realmente revelador, en un sentido puramente descriptivo, del fenómeno de financiarización del suelo urbano en Santiago. No obstante, y consistente con lo previsible, también es alta la participación del rubro de "actividades inmobiliarias, empresariales y de alquiler", las cuales compran un total de 2.023 terrenos, representativos del $31 \%$ de las adquisiciones. Que estos dos rubros sumen un 70\% y sean ampliamente los más participativos de este mercado puede no ser tan novedoso, pero que la dimensión que adquieran las entidades financieras sea tan alta, superior a las actividades inmobiliarias naturalmente vinculadas a las adquisiciones y enajenaciones de bienes raíces, parece un hallazgo que hasta la fecha no había sido explorado.

\section{FIGURA I $\mid$ Resumen de adquisiciones por rubro informado en sII}

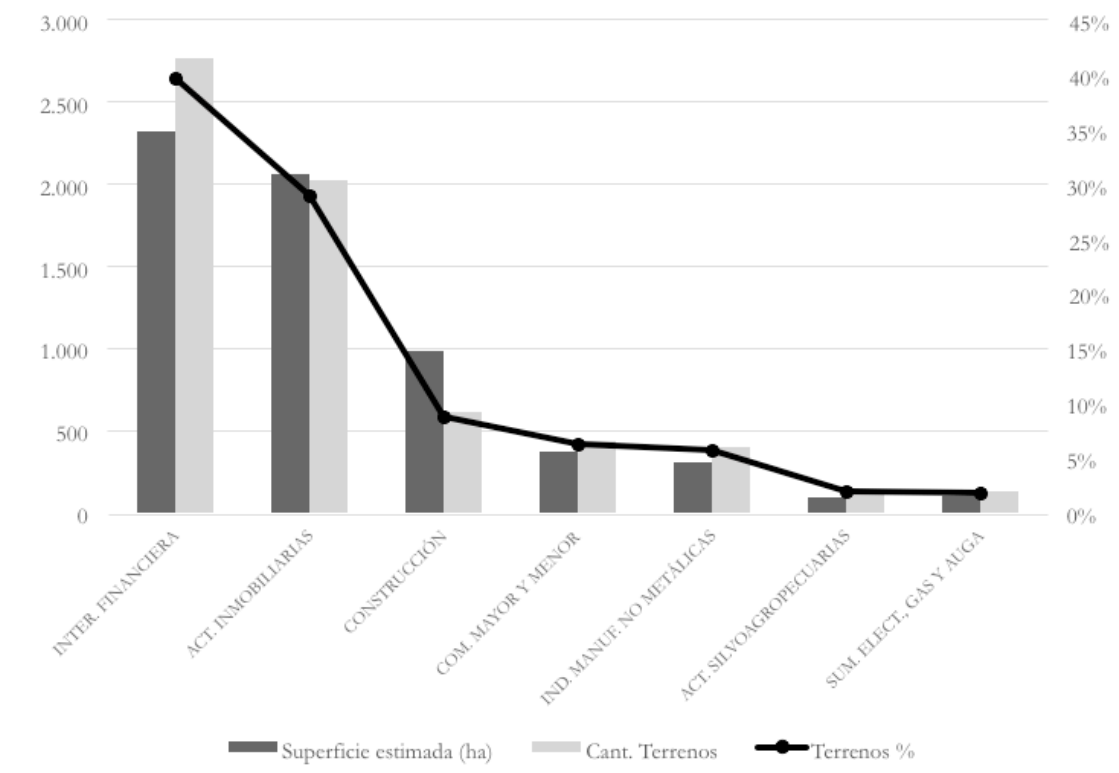

FUENTE ELABORACIÓN PROPIA CON DATOS DEL CBRS Y EL SII (2OI6)

Dentro del rubro financiero, las entidades que más compran terrenos corresponden a sociedades de inversión altamente fragmentadas

Al desagregar los datos a nivel de actividades económicas, se observa que la mayor cantidad de adquisiciones de terrenos las efectúan "sociedades de inversión y rentistas de capitales mobiliarios", es decir, inversionistas que compran bienes raíces sin orientarlos a un uso productivo (incluyendo producción inmobiliaria) y cuyo 
interés es únicamente el beneficio obtenido del flujo de renta o el diferencial de precio de venta (lo que comúnmente se denomina "plusvalía"). Estas sociedades suman un total de 1.674 terrenos comprados en el período, con una superficie estimada total de 1.375 hectáreas transadas (flujo, no stock) y una inversión total de 38,3 millones de Uf.

En estas sociedades de inversión puede verse un nivel bastante bajo de concentración de los agentes, con un total de 662 razones sociales distintas que adquieren suelo en Santiago, a razón de 2,5 terrenos cada una. Por ende, si bien es la principal categoría de intermediación financiera sobre el mercado de terrenos, el nivel de fragmentación de los agentes es radicalmente distinto a lo que sucede con las otras entidades que participan de este mercado.

FIgURA 2 Participación de principales Sociedades de Inversión y Renta de Capitales Mobiliarios en mercado del suelo de Santiago, período 2010-2015

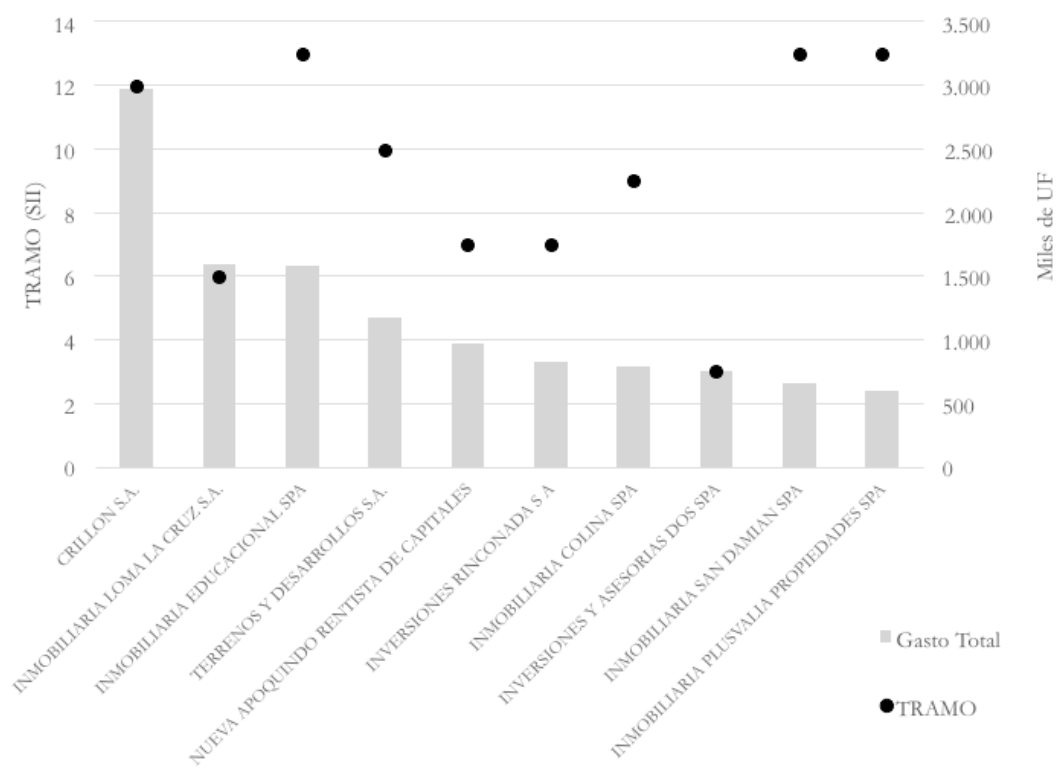

FUENTE ELABORACIÓN PROPIA CON DATOS DE CBRS Y SII (2OI6)

El gráfico muestra las sociedades con mayor inversión en terrenos en el período, y el respectivo tramo de facturación económica. En términos de capital propio positivo hay muchas disparidades y algunas sociedades están sin información, por lo que se ha utilizado alternativamente el dato de "tramo". Así, es posible dar cuenta de que la mitad de estas sociedades son consideradas grandes empresas, por sobre las 100.000 uf de facturación anual. Y tres de estas sociedades pertenecen al tramo 13, que corresponde a las empresas más grandes del país, con facturación por sobre 1.000 .000 uf anual. 
Todas estas sociedades invierten más de 500.000 uf en terrenos en el período considerado, en un rango que va hasta las 1.600 .000 uf, sin contar el caso de Crillón s.A., la cual presenta una inversión total sustantivamente mayor, que llega a las 3.000.000 Uf, en sus 19,6 hectáreas adquiridas. Sin embargo, esta sociedad, perteneciente a la familia Cousiño, tiene rasgos particulares que impiden extender su comportamiento al conjunto de estas sociedades. Crillón es una figura jurídica, entre otras, como Terra Australis, utilizada para administrar los terrenos que han dado forma a los proyectos inmobiliarios operados sobre la Viña Cousiño Macul en la comuna de Peñalolén.

También es interesante notar algunos casos, como el de "Terrenos y Desarrollos" y el de "Inmobiliaria Plusvalía Propiedades Spa". En ambos se trata de sociedades para captar plusvalías creadas por bancos, BCI y Santander, respectivamente, con participación controladora del $100 \%$. De esta manera, pese a la cantidad de sociedades de este tipo y su aparente fragmentación, huelga considerar en un futuro trabajo sus niveles de dependencia y articulación vertical con grandes desarrolladores, bancos y fondos de inversión inmobiliaria. Esto es particularmente importante para este grupo de sociedades, ya que: (i) se trata de agentes que operan por proyecto; (ii) su carácter es de "renta de capitales mobiliarios", es decir, acciones y derechos; y además, (iii) en el estrato de mayor participación se exhibe una relación societaria con grupos inmobiliarios y bancos.

Existe un núcleo de capital financiero altamente centralizado compuesto por bancos y compañías de seguros de vida, aunque con una participación sustantivamente menor en comparación con las sociedades de inversión

Además de las sociedades de inversión, que corresponden a agentes que no tienen por objeto la transformación del espacio físico, sino la absorción de flujos de renta derivados de procesos externos de valorización de los terrenos, es posible observar otro grupo de agentes de intermediación financiera cuantitativamente menos representativos, pero cualitativamente muy relevantes. A saber, en un $14 \%$, las transacciones en el período (998 terrenos, con una superficie estimada de 912 hectáreas) han sido adquisiciones de bancos y compañías de seguros de vida, ambas con una participación casi idéntica en volumen de compras y superficie total adquirida.

A diferencia de los inversionistas, en este caso las adquisiciones corresponden a operaciones de financiamiento a otras empresas y sociedades, por lo que su componente de especulación inmobiliaria pasa a un segundo orden frente a lo que representa la ganancia en la forma de interés sobre el capital invertido. Por eso, este conjunto de bancos y aseguradoras representa un segmento aparte en el análisis, y se ha observado en casos particulares que el modo de operación de estas entidades consiste en la ejecución de contratos de leasing o lease-back a empresas y desarrolladores inmobiliarios.

En el caso de las aseguradoras, estas han emergido como importantes agentes de intermediación de terrenos, con un total de 508 adquisiciones entre 2010 y 2015. En cuanto a la inversión total realizada en el período, se observa que seis de las catorce compañías de seguros de vida han invertido más de un millón de UF, llegando a casi dos millones de uf en los casos de Euroamérica y Penta Vida, que 
además son las que tienen la mayor cantidad de adquisiciones, con un 60\% del total. Considérese que, para el conjunto de estas compañías, se trata de empresas del rango de tamaño más grande en la categorización de empresas en el país, todas dentro del tramo de facturación del sil correspondiente a "gran empresa" de más de 1.000.000 uf de facturación y con capital propio positivo (CPP) declarado de más de 45 mil millones de pesos, llegando en el caso de Metlife Chile a los 370 mil millones de pesos.

En relación con lo anterior, es interesante notar que la inversión total en terrenos no parece ser correlativa a la dimensión de las empresas en términos de capital y facturación. Al ordenar descendentemente las compañías según cPP declarado, no se observa una correlación directa entre el tamaño de las compañías y sus volúmenes de adquisición de terrenos. Metlife, una de las compañías líderes en el rubro asegurador a nivel mundial, aparece con el capital positivo propio más alto y, sin embargo, muy por debajo en inversión en terrenos respecto a compañías como Euroamérica o Penta Vida, de carácter local. Este dato, corroborado con posteriores análisis, puede resultar de gran interés para ponderar el peso relativo que tienen las adquisiciones de terrenos en el total de activos de las compañías mencionadas.

\section{FIgURA 3 Participación de compañías de seguros de vida (Csv) en mercado del suelo de Santiago. Relación entre capital al 2015 e inversión en terrenos en período 2010-2015}

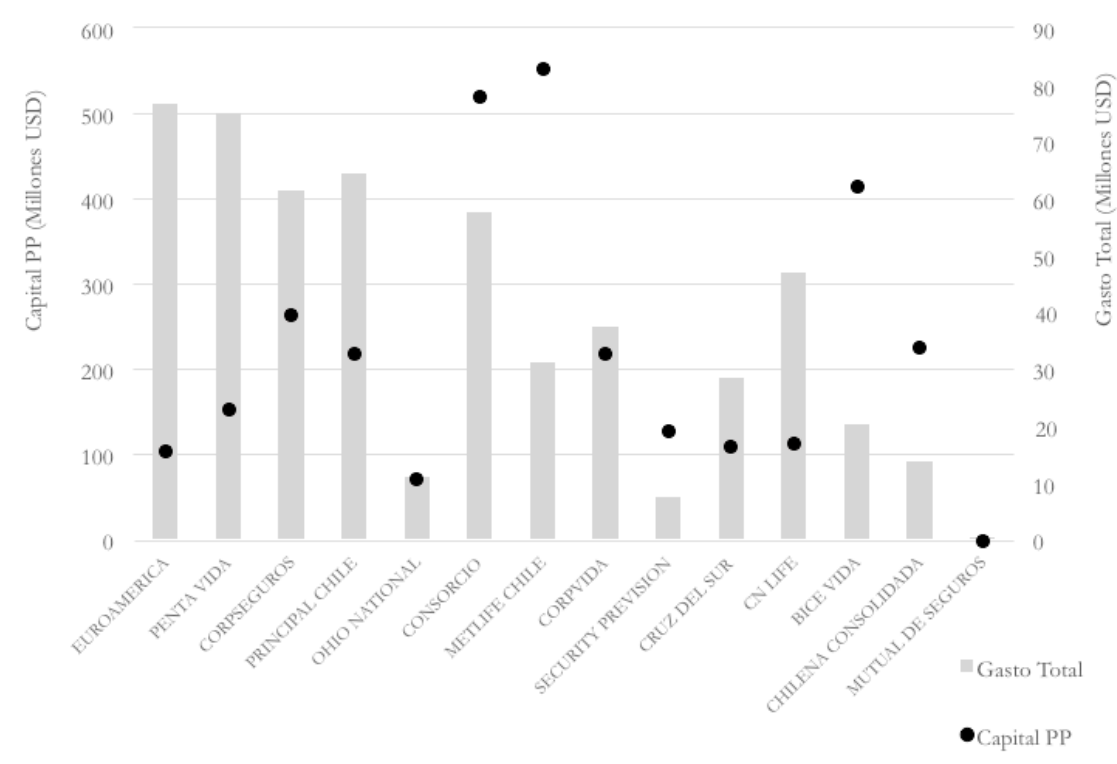

FUENTE ELABORACIÓN PROPIA CON DATOS DE CBRS Y SII (2OI6) 
FIgURA 4 | Participación de bancos en mercado del suelo de Santiago. Relación entre capital al 2015 e inversión en terrenos en período 2010-2015

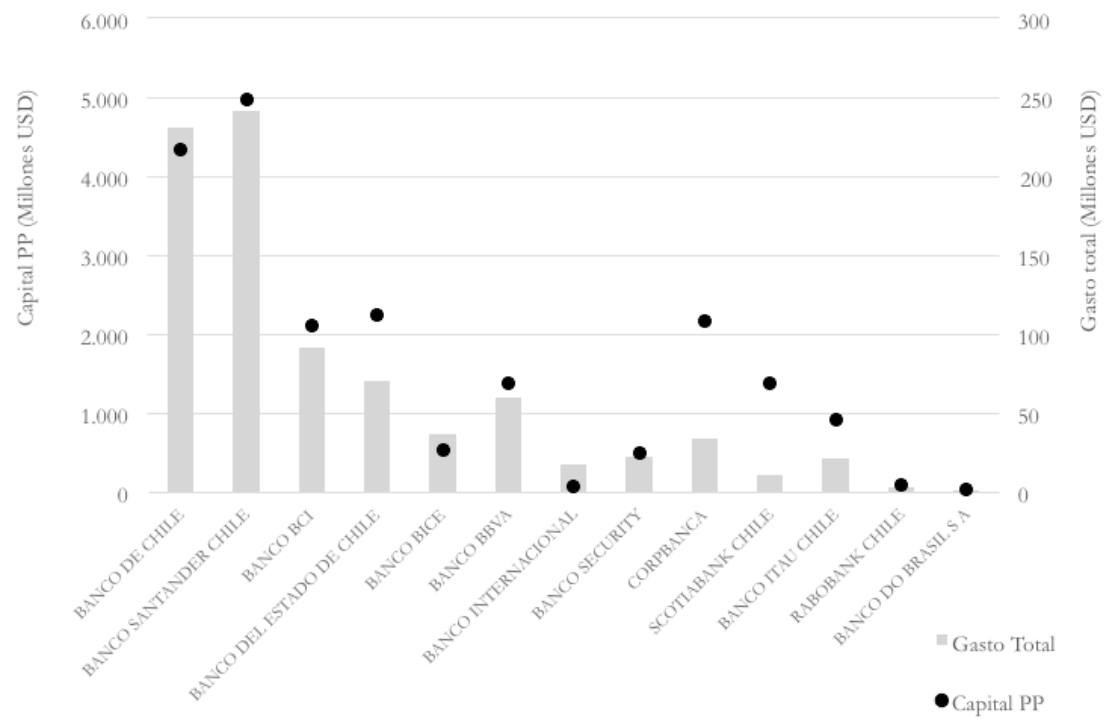

FUENTE

ELABORACIÓN PROPIA CON DATOS DE CBRS Y SII (2OI6)

Por su parte, los bancos han comprado 493 terrenos en el período, con una superficie estimada de 501 hectáreas. A diferencia de las aseguradoras, en este caso se observa una concentración mucho más alta de las operaciones en algunos pocos agentes. Básicamente dos son los bancos que concentran esta actividad, que corresponden a los dos bancos más grandes que operan en el país: Banco de Chile y Banco Santander Chile. Entre ambos agregan poco más del 50\% de los terrenos adquiridos, y el 38\% de las quinientas hectáreas compradas por el sector bancario en su conjunto, constituyéndose en grandes terratenientes del Área Metropolitana de Santiago. ${ }^{13}$ Esto a pesar de que la Ley de Bancos les obligue a comprar terrenos solo mediante contratos de leasing, dificultando entonces que los terrenos se transformen en activos de valorización inmobiliaria propiamente tal.

Las grandes empresas son las que más invierten en el mercado de terrenos de Santiago, representadas por el capital bancario y asegurador

Las empresas más grandes del país, particularmente las del tramo 13 de mayor nivel de facturación a nivel nacional, son las principales compradoras de terrenos en Santiago. Tienen una participación muy por encima del resto, ascendiendo a un total de gasto en adquisición de terrenos de 59 millones de uf en el período. Esto considerando además que el rango de gran empresa, en sus cuatro tramos, es el que

13 Evidentemente esto debe corroborarse observando el total de sus activos y no solo aquellos adquiridos en el período, pero por la magnitud alcanzada es aceptable una denominación de este tipo. 
tiene menor cantidad de empresas en el país según el sir. ${ }^{14} \mathrm{El}$ ensanchamiento de empresas en la medida en que baja su tamaño permite dar mayor relevancia a los datos que aquí se exponen.

Sin embargo, es muy importante constatar que los datos muestran un comportamiento fuertemente aleatorio entre los tramos, donde se torna muy difícil establecer una relación entre tamaño de las empresas y nivel de participación en el mercado de terrenos, medido en cantidad de transacciones, superficie y gasto. Solo puede constatarse que el conjunto de las grandes empresas -en particular el tramo 13, también denominado por el sil como gran empresa de cuarto rango-, tuvo una participación destacada en el mercado de terrenos en Santiago entre 2010-2015, con una superficie estimada de 2.182 hectáreas transadas y un gasto de 96 millones de UF (datos agregados para el conjunto de los cuatro tramos de grandes empresas consignadas por el siI).

TABLA I | Adquisiciones según tamaños de empresas y tramos

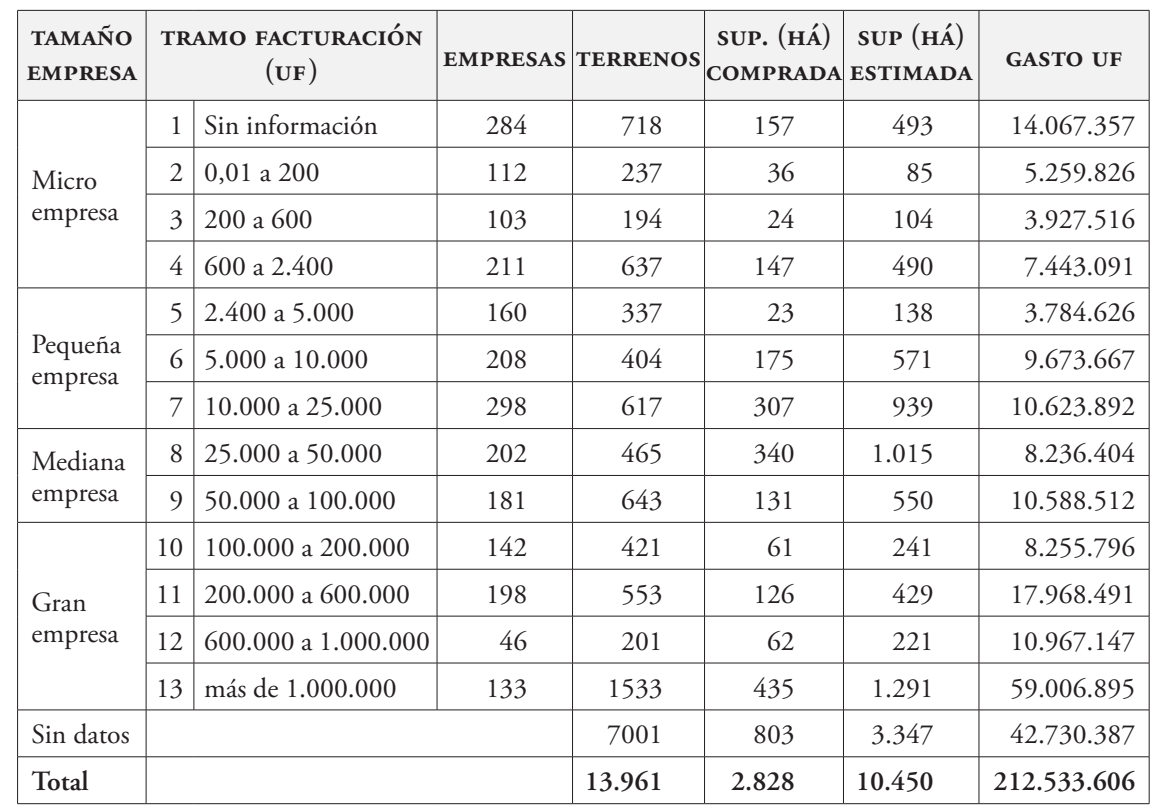

FUENTE ELABORACIÓN PROPIA CON DATOS DE CBRS Y SII (20I6)

Por otra parte, si se observa al interior del tramo 13, es decir, las empresas y sociedades más grandes del país, se identifica que aquellas con mayores montos invertidos en adquisición de terrenos son los bancos y las compañías de seguros de vida, reforzando el resultado recientemente expuesto. Es decir, son estas entidades financieras las que están expresándose en los resultados obtenidos de participación de las grandes empresas. A este grupo, con inversiones totales en el período entre los

$14 \mathrm{Al} 2014$ se identifica un total de 13.979 grandes empresas en estos cuatro tramos, sobre un total de 895.836 empresas individualizadas en el país, considerando creación y destrucción de las mismas. 
1 y 6,3 millones de UF, se agregan algunas sociedades inmobiliarias, pero básicamente orientadas a la renta de inmuebles. Aparece aquí una de las empresas líder en centros logísticos y almacenamiento, como es Bodegas San Francisco Limitada, y la sociedad inmobiliaria denominada Inmobiliaria Educacional Spa, destinada a arrendar establecimientos educacionales del grupo Laureate (grupo norteamericano dueño de Universidad Las Américas, Universidad Andrés Bello, Universidad Viña del Mar e Instituto AIEP). Un tercer agente que aparece con un importante nivel de inversión es la Compañía de Cervecerías Unidas (CCU), aunque se trata de operaciones puntuales de alta envergadura en adquisición de terrenos para uso propio de su planta industrial. El resto de las sociedades corresponde a bancos y compañías de seguros de vida.

FIGURA 5 Gasto en adquisición de terrenos y CPP de las grandes empresas del país (tramo 13, rango 4), con rango de inversión total entre 1 y 7 millones de UF en período enero 2010-abril 2015

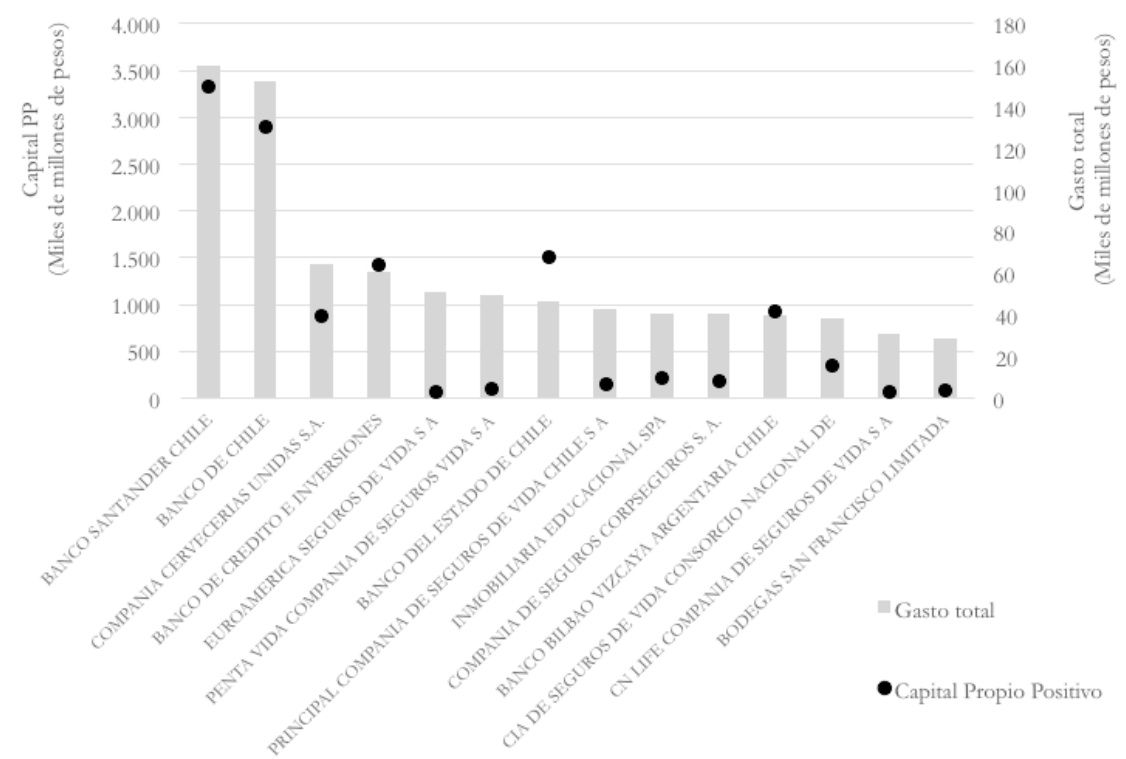

FUENTE ELABORACIÓN PROPIA CON DATOS DE CBRS Y SII (20I6)

Luego se ha identificado un segundo orden de empresas que han invertido entre 500.000 y 1 millón de uf en el período, correspondientes también a grandes empresas (tramo 13, igual que las anteriores). Aquí es posible notar una presencia dominante de las sociedades inmobiliarias, desarrolladores y rentistas más importantes del país. En el caso de las inmobiliarias, como funcionan con muchas razones sociales (a diferencia de los bancos y compañías de seguros), se distorsionan aún más los resultados y, en especial, el valor de referencia de CPP. Por ello aparecen algunas sociedades como Socovesa Santiago s.A., donde se registra solo el CPP de esa filial, que para efectos de un análisis más depurado debiese reclasificarse en una 
categoría más amplia que incorpore todas las distintas filiales de este grupo inmobiliario matriz.

No obstante lo anterior, referido a las limitaciones metodológicas de este trabajo, se puede observar que en este segundo orden, entre 500.000 y 1.000 .000 uf, ya comienzan a aparecer importantes inmobiliarias que dan cuenta de su aporte específico a la categoría de grandes empresas. Además del caso de Socovesa, aparece en este segmento una de las principales inmobiliarias del grupo Paz Corp (Inmobiliaria Paz Spa), Simonetti Inmobiliaria, Inmobiliaria Py y Almagro. Es decir, un segmento de las principales inmobiliarias promotoras de vivienda en casa y departamento. Además, en el rubro de la construcción aparece la Constructora Moller y Pérez Cotapos. El resto está compuesto por algunos bancos, compañías de seguros y sociedades inmobiliarias de renta ligadas al retaily la distribución, como Socofar (Inmobiliaria ligada a Farmacias Cruz Verde) y Sociedad de Rentas Falabella (adquiere solo dos terrenos, pero de muy alto precio, para localización de Multitiendas Falabella).

FIGURA 6 | Gasto en adquisición de terrenos y CPP de las grandes empresas del país (tramo 13, rango 4), con rango de inversión total entre 500.000 y 1 millón de uf en período enero 2010-abril 2015

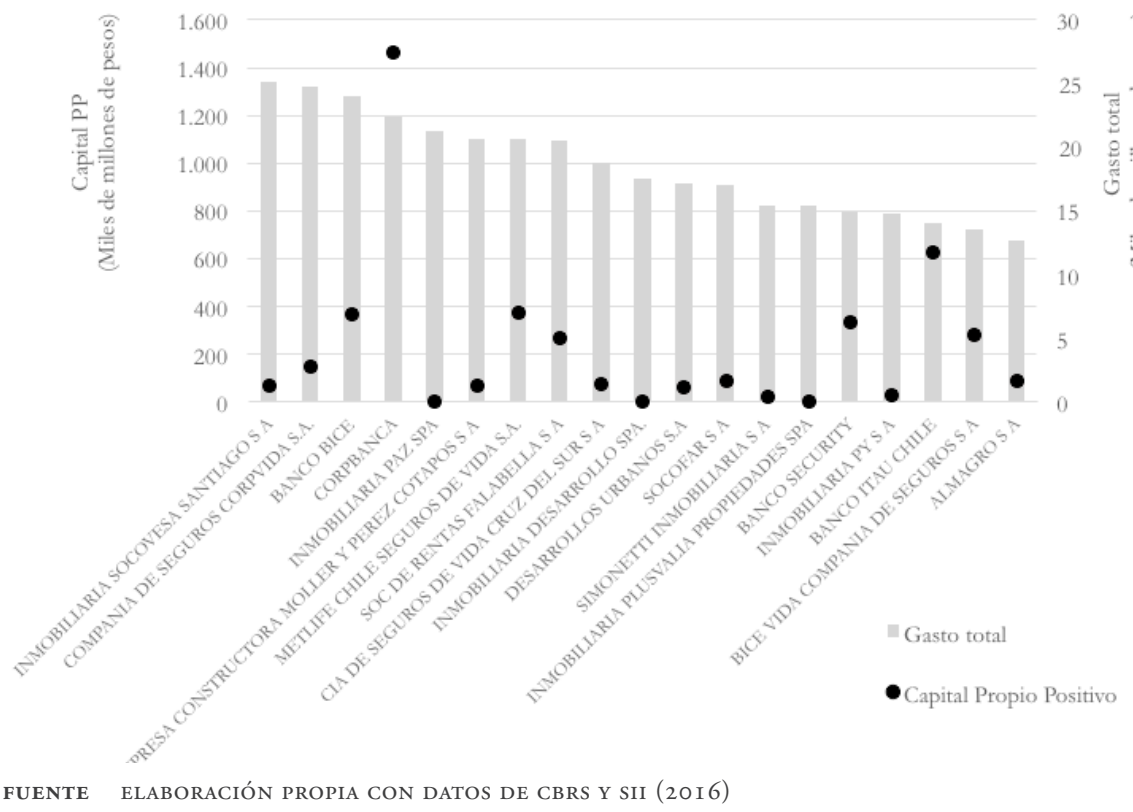

Lo interesante de este segmento de grandes empresas (tramo 13) con inversiones entre las 500.000-1.000.000 uf es que, a diferencia del segmento anterior, logra una predominancia de desarrolladores inmobiliarios y sociedades de renta inmobiliaria, y en todos los casos se trata de los principales agentes del mercado habitacional en Santiago. Por su parte, cabe recordar que este ejercicio cuenta solo con el tramo de las empresas más grandes del país, lo que no quiere decir que no haya empresas 
más pequeñas con mayor participación en el mercado de terrenos. Cualquiera sea el caso, la observación en particular de estos datos permite dimensionar la participación de las grandes empresas del país y confirmar su naturaleza: en primer lugar financiera, y luego inmobiliaria. Es una forma más de expresar este resultado convergente del presente trabajo.

\section{Conclusiones y reflexión final}

Se ha identificado una participación protagónica de sociedades de capital inmobiliario y financiero en el mercado de terrenos de Santiago, con 70\% de las transacciones de sociedades (excluidas las personas naturales). Luego, lo que se ha encontrado es una participación relativa muy alta de actividades de intermediación financiera donde, sorprendentemente, el volumen agregado de transacciones, superficies e inversión en terrenos del conjunto de estos capitales logra ser superior al rubro de actividades inmobiliarias y constructoras. Este es uno de los hallazgos fundamentales de este trabajo y queda al pendiente una explicación exhaustiva al respecto.

Dentro del rubro de intermediación financiera es posible notar una diferencia entre dos formas de capital substantivamente distintas. Por una parte, existe un robusto sector de la economía financiera compuesto básicamente por bancos y aseguradoras, es decir, actividades de intermediación monetaria y de administración de fondos de seguridad social privados (compañías de seguros de vida), los cuales presentan características, al parecer, propias de agentes financieros, intermediando a interés las compras de los agentes inmobiliarios con uso efectivo del suelo. En primera instancia, debe reconocerse que la adquisición directa de tierras por entidades financieras y bancarias, que además forman parte del grupo de más grandes empresas del país, revela una mayor capitalización en el sector, donde el financiamiento de los terrenos no corresponde exclusivamente a los desarrolladores con sus capitales propios -sean privados, de holdings mayores o del mercado bursátil-, sino que acuden a su colaboración otras entidades "no-inmobiliarias" con un carácter mayormente financiero (en un sentido amplio, incluyendo a las sociedades de inversión pasiva). El entramado de agentes financiero-inmobiliarios puede parecer obvio, pero no lo es necesariamente el hecho de que esto se exprese en la compra directa de terrenos.

Huelga indagar particularmente en los instrumentos de leasing y lease-back que permiten a estas sociedades adquirir terrenos para los desarrolladores con compromiso de venta, estableciendo un canon de arriendo teóricamente fijado por el interés sobre el capital invertido. Estos instrumentos son los que permiten al sector bancario y asegurador comprar tierras a mediano y largo plazo, aunque con algunas diferencias. Como el principal interés de los bancos (por el lado de la oferta) está puesto en las líneas de crédito a la construcción, es previsible que opten por operaciones de leasing de terrenos a menor plazo asociados a la construcción. Por su parte, si bien las compañías de seguros no están obligadas a adquirir mediante leasing o lease-back como en el caso de los bancos, la observación de algunas operaciones permite deducir que estos son instrumentos altamente empleados. Por la lógica del 
rubro asegurador, es previsible que se den operaciones a más largo plazo vinculadas al pago de las rentas vitalicias del sector, y no necesariamente a la articulación con financiamientos a la construcción. Esta puede ser una clave analítica para diferenciar ambas entidades, a pesar de tener un comportamiento similar como capital a interés incorporado al mercado del suelo.

Por otra parte, aparecen también capitales que tienen el carácter de "especulativos" vinculados a sociedades de inversión. A diferencia de los capitales financieros propiamente tales, estos capitales que aparecen bajo la forma de "sociedades de inversión y renta” están orientados a la obtención de plusvalías por la compraventa de las propiedades, pero sin funcionar como financiamiento al terreno de algún desarrollador. En este tipo de prácticas, propias de lógicas especulativas, lo que ocurre es que la compra de terrenos se genera producto de una mayor expectativa de alza en los precios de los mismos, como consecuencia de los eventuales precios finales en el mercado inmobiliario. Esta lógica está fundamentada, básicamente, en la expectativa de la disponibilidad de ingresos de los consumidores finales de los productos inmobiliarios; más específicamente, de la capacidad de pago y endeudamiento de los hogares. La teoría de la renta de la tierra se basa en esa determinante para explicar los precios de los terrenos, lo que los vuelve particularmente sensibles a los ingresos. Bajo esta lógica se desvincula la relación entre adquisición de terrenos y volumen de producción en el mercado inmobiliario, tornando más dinámica la variable relativa a las expectativas de precios finales al consumidor. Lo cierto es que la alta volatilidad de los precios finales de este tipo de mercancías, desvinculadas de su costo de producción y reposición, hace a estos capitales más expuestos al riesgo -y a las burbujas inmobiliarias-, a diferencia del caso de los bancos y aseguradoras.

Este trabajo ha tenido por finalidad la difusión de un conjunto de datos que permiten estimular hipótesis, planteamientos y nuevas preguntas sobre cómo ha ido ingresando el capital financiero al mercado del suelo en Santiago de Chile en los últimos años. Como ninguna serie de datos puede, por sí misma, habilitar al investigador a sacar conclusiones, este trabajo se ha remitido a estimular la formulación de preguntas que emerjan del contacto con la realidad empírica. En este sentido, los datos que se han presentado permiten interrogar algunas cuestiones de orden estructural y otras relativas a las prácticas concretas de los agentes. En relación con estos, ¿bajo qué condiciones los bancos prefieren comprar terrenos para ponerlos a disposición de los desarrolladores en vez de financiar con préstamos? ¿Por qué las compañías de seguros de vida invierten directamente en terrenos para los desarrolladores y no se dedican a la compra de bonos y acciones de estos o de fondos de inversión inmobiliaria? ¿Qué nivel de integración y/o relación societaria tienen las sociedades de inversión con los desarrolladores inmobiliarios, o se trata de entidades independientes que comercializan los terrenos en un mercado abierto expuesto a mayor riesgo? Y, en síntesis, ¿por qué los desarrolladores estarían externalizando el bodegaje de terrenos en este entramado de entidades financieras y de inversión?

Además de estas preguntas que emergen de la investigación empírica, se mantienen latentes algunas preguntas de orden estructural que debiesen ser incorporadas en estudios más comprensivos sobre la materia. Por ejemplo, ¿qué función cumple el capital financiero que ingresa a la adquisición de terrenos en relación 
con las dinámicas capitalistas de producción inmobiliaria, considerando la fuerte componente rentista de este sector? ¿Se orienta a financiar el desarrollo capitalista de vivienda, a especular con la valorización de activos o eventualmente a establecer reservas de valor frente a coyunturas en la depreciación del dinero o las monedas? ¿Cómo relacionar correctamente estos grandes volúmenes de inversión a la financiarización de la economía capitalista mundial, considerando que operan compañías de capital extranjero e inversionistas de alcance global? La amplia literatura internacional ha avanzado en parte de estos asuntos, pero la escasa disponibilidad de datos en el mercado inmobiliario dificulta la comprobación de las hipótesis teóricas. Al menos a nivel local, este trabajo espera haber aportado a subsanar ese déficit de evidencia empírica.

Por último, se considera necesario continuar el estudio de las relaciones entre producción inmobiliaria capitalista, mercados del suelo y financiarización de la economía, dado que las lógicas de valorización del capital imponen dinámicas estructurantes para la ciudad y límites reales a la planificación urbana. La formación de una fracción de la clase capitalista especializada en la compra masiva de tierras para desarrollo inmobiliario puede estar explicando, en buena parte, la escasez artificial de suelo urbano y el alza en el precio del mismo, con las consecuencias que ello tiene en la exclusión social de los segmentos más pobres de la población que actualmente no tienen acceso a suelo urbanizado y bien localizado en el Área Metropolitana de Santiago.

\section{Agradecimientos}

Agradecimientos al Proyecto Fondecyt 1141157 "Nueva Geografía de la Urbanización y Vulnerabilidad Socio-territorial. ¿Hacia la configuración de una Región Urbana en Santiago de Chile?”, por el apoyo y financiamiento entregado a este trabajo; y a Transsa Limitada por facilitar el acceso a la base de datos de transacciones.

\section{Referencias bibliográficas}

Asociación AfP [Administradoras de Fondos de Pensiones] Chile (2012). Publicaciones / Estudios 2012 [en línea]. https://www.aafp.cl/publicaciones/estudios/

Alarcón, A., Demaestri, E. \& Piedrabuena, B. (2014). Financiamiento de la vivienda en Chile. Nota Técnica IDB-TN-693. Banco Interamericano de Desarrollo. http://bit.ly/2jlr6Cd

Aglietta, M. (1979). Regulación y crisis del capitalismo. La experiencia de los Estados Unidos. Madrid: Siglo Xxi.

Bellamy-Foster, J. (2010). The financialization of accumulation. Monthly Review, (62)5. https:// monthlyreview.org/2010/10/01/the-financialization-of-accumulation/

Cattaneo, R. (2011). Los fondos de inversión inmobiliaria y la producción privada de vivienda nueva en Santiago de Chile. ¿ Un nuevo paso hacia la financiarización de la ciudad? EURE, 37(112), 5-22. https://dx.doi.org/10.4067/S0250-71612011000300001 
Cheetham, R. (1971). El sector privado de la construcción: patrón de dominación. EURE, 1(3), 125-148. https://repositorio.uc.cl/bitstream/handle/11534/4033/000389426. pdf?sequence $=1$

Chesnais, F. (2003). La teoría del régimen de acumulación financiarizado: contenido, alcance e interrogantes. Revista de Economía Crítica, n. 1 (abril), 37-72. http://www. revistaeconomiacritica.org/sites/default/files/revistas/n1/4_teoria_regimen.pdf

Daher, A. (1995). El efecto concentrador de los fondos inmobiliarios. Revista de Ciencias Sociales, Universidad de Costa de Rica, (68), 17-32.

Daher, A. (2013). El sector inmobiliario y las crisis económicas. EURE, 39(118), 47-76. https://dx.doi.org/10.4067/S0250-71612013000300003

De Mattos, C. (2013.). Globalización financiera, dinámica inmobiliaria y mercantilización del desarrollo urbano. Documento de trabajo interno, Pontificia Universidad Católica de Chile, Santiago de Chile.

De Mattos, C. (2016). Financiarización, valorización inmobiliaria del capital y mercantilización de la metamorfosis urbana. Revista Sociologías (Porto Alegre), (42), 24-52. https:// dx.doi.org/10.1590/15174522-018004202

Fondo Monetario Internacional (FMI) [International Monetary Fund (IMF)]. (2015). Understanding residential real estate in China. IMF Working paper 15/84, por M. Chivakul, W. R. Lam, X. Liu, W. Maliszewski, \& A. Schipke. http://bit.ly/2AwVRO4

Harvey, D. (1974). Class-monopoly rent, finance capital and urban revolution. Regional Studies, 8(3-4), 239-255. https://doi.org/10.1080/09595237400185251

Harvey, D. (2012). Ciudades rebeldes. Del derecho de la ciudad a la revolución urbana. Madrid: Akal.

Hudson, M. (2010). The transition from industrial capitalism to a financialized bubble economy. Working paper n. 627, Levy Economics Institute of Bard College. http://www. levyinstitute.org/pubs/wp_627.pdf

Husson, M. (2010). El debate sobre la tasa de beneficio. Trad. M. de la Fuente de «Le débat sur le taux de profit», Inprecor, 562-563, junio-julio 2010. Versión en español en http:// www.vientosur.info/documentos/Tasa\%20beneficio-Husson2.pdf. Texto original en http://hussonet.free.fr/debaprof.pdf

Lapavitsas, C. (2010). Financialisation and capitalism accumulation: structural accounts of the crisis of 2007-9. Political Economy Quarterly 47(1), 42-55. https://doi.org/10.20667/ peq.47.1_42

Lapavitsas, C. (2016). Beneficios sin producción. Cómo nos explotan las finanzas. Madrid: Traficantes de Sueños. https://www.traficantes.net/sites/default/files/pdfs/PC10_ beneficios_web.pdf

Pablo Trivelli \& Compañía Limitada (2010). Urban structure, land markets and social housing in Santiago, Chile. Working papper BID. Santiago de Chile: Banco Interamericano de Desarrollo. Versión digitalizada en http://www.cafedelasciudades.com.ar/ imagenes109/012411\%20Informe\%20final_PTrivelli.pdf

Sabatini, F. (1981). ¿Por qué aumentan los precios del suelo en Santiago? ¿Representa ello mayores costos de construcción o mayores ganancias inmobiliarias? Documento de Trabajo $\mathrm{N}^{\circ}$ 120. Santiago de Chile: Centro Interdisciplinario de Desarrollo Urbano-Instituto de Planificación Urbana (Cidu-IPu), Pontificia Universidad Católica de Chile. 
Sabatini, F. \& Donoso, F. (1979). La renta de la tierra en el desarrollo residencial reciente de Santiago. Santiago de Chile: Centro Interdisciplinario de Desarrollo Urbano-Instituto de Planificación Urbana (cidu-IPU). P. Univ. Católica de Chile

Sabatini, F. \& Donoso, F. (1980). Algunas hipótesis sobre la importancia de la renta de la tierra en el desarrollo reciente de Santiago. Documento de Trabajo N 114, Santiago de Chile: Centro Interdisciplinario de Desarrollo Urbano-Instituto de Planificación Urbana (CIDU-IPU). P. Univ. Católica de Chile.

Theurillat, T. (2012). The negotiated city: between financialisation and sustainability. Research Group on Territorial Economics (GRET). University of Neuchâtel, Switzerland. http://www.nfp54.ch/files/nxt_projects_87/19_01_2010_02_59_49NegociatedCity_2009.pdf

Topalov, C. (1979.). La urbanización capitalista. México, D.F.: Edicol.

Zegers, F. (2014). Financiarización, mecanismos de financiamiento de vivienda y configuración territorial metropolitana de Santiago, 1990-2011. Tesis para obtener el grado académico de Magíster en Desarrollo Urbano, Instituto de Estudios Urbanos y Territoriales, Pontificia Universidad Católica de Chile. Santiago de Chile. http://estudiosurbanos. uc.cl/images/tesis/2014/MDU_Francisca_Zegers.pdf 